\section{Psychoedukation als psychosoziale Intervention in der Drogentherapie}

Zusammenfassung: Im Rahmen der Ausschreibung des Modellversuchs zu heroingestützten Therapieansätzen drängte sich erneut die Frage nach den Standards psychosozialer Behandlung auf.

Aus unserer Sicht ist es hierzu nötig, einen Überblick über den Stand der Forschung zu erstellen und eine Neueinordnung vorzunehmen. Ein Ziel ist es, zu mehr Klarheit zu kommen, welche Interventionen in Zukunft in der Drogenhilfe standardisiert angewandt werden können.

Aufgrund einer längerfristigen Auseinandersetzung mit dem Thema psychotherapeutischer Interventionen in der Drogentherapie wollen wir speziell einige Überlegungen zur Psychoedukation zur Diskussion stellen, die aus Sicht der Autoren eine Variante psychosozialer Intervention darstellen kann.

Schlüsselwörter: Heroingestützte Behandlung - Psychosoziale Interventionen - Psychoedukation - Drogentherapie

Psychoeducation as a Psychosocial Therapy in Addiction Treatment: In the context of planning the proposed trial on heroin assisted treatment (heroin maintenance), questions concerning the standards of concomitant psychosocial treatment came up again.

From our point of view, it is necessary, therefore, to review and re-evaluate psychosocial intervention in view of the latest results of research, with the aim to assess which interventions are best suited for standardised therapies in addiction treatment.

Following longterm reflections on the issue of psychotherapeutic interventions in drug therapy, we bring up for discussion some views concerning psychoeducation which might become an additional psychosocial treatment variant in addiction treatment.

Key words: Heroin Perscription - Psychosocial Interventions - Psychoeducation - Drug therapy

\section{Vorbemerkung}

Im Rahmen der Ausschreibung des Modellversuchs zu heroingestützten Therapieansätzen wurden auch die Fragen psychosozialer Betreuung für Drogenabhängige in der Diskussion aktualisiert. Dabei stellten sich unter anderem die Fra-

Suchttherapie 2000; $1: 83-88$

(c) Georg Thieme Verlag Stuttgart · New York

ISSN 1439-9903
Michael Krausz, Georg Farnbacher

Zentrum für Interdisziplinäre Suchtforschung (ZIS) der Universität Hamburg

gen, welche Rolle die Seite der psychosozialen Intervention im Verhältnis zu den pharmakologischen Interventionen zu spielen hat, welche Standards verbreitet sind und wie der Stand der Forschung in diesem Therapiefeld ist. Eine wesentliche Frage der Gutachter der WHO, die für die Beurteilung der Effektivität der Heroinsubstitution bzw. der Originalstoffvergabe im Rahmen integrierter Programme in der Schweiz von hoher Bedeutung war, zielte genau auf den Stellenwert des psychosozialen Teils an den Untersuchungsergebnissen: War nicht die intensive Begleitbetreuung das Erfolgsgeheimnis der Schweizer Programme?

Neben der Reflexion dieser Betreuung und der Klärung des Foschungsstandes auf heutigem Niveau sind Bewertungen erforderlich, welche Interventionen auf welche Weise für medikamentengestützte Therapien nutzbar zu machen sind.

Ein Vorschlag der Hamburger Gruppe im Rahmen des geplanten Modellprojekts Heroin geht daher in die Richtung, zwei unterschiedliche Verfahren als Methoden der psychosozialen Begleitung anzuwenden und zu vergleichen:

- Das Case-Management als ein strukturiertes, individuell begleitendes und nachgehendes Konzept der Betreuung mit hoher Kontaktdichte sowie

- die Kombination aus Drogenberatung und Psychoedukation, d.h. durch eine fakultative Nutzung örtlicher Drogenberatung (Komm-Struktur) einerseits und einem zusätzlichen Psychoedukationsprogramm von 12 bis 15 Sitzungen über drei Monate hinweg in einem gruppentherapeutischen Setting.

Durch einen systematischen Vergleich zweier standardisierter psychosozialer Begleitbehandlungen - Case-Management versus Drogenberatung mit Psychoedukation - können Therapieeffekte - im Rahmen der Vergabe von i.v. Heroin im Vergleich zu oralem Methadon - unterschiedlicher Settings evaluiert werden.

\section{Psychosoziale Interventionen in der Behandlung Drogenabhängiger}

Eine zeitgemäße Therapie drogenabhängiger Menschen muss pharmakologische wie psychotherapeutische, pädagogische wie sozialtherapeutische Interventionen personenspezifisch berücksichtigen. Sowohl in den vorliegenden Standards beispielsweise zur Methadonsubstitution [1,2,3] als auch aus den Ergebnissen der Therapieforschung [4, 5, 6,7] ergibt sich die Notwendigkeit einer qualifizierten Bereitstellung psychosozialer Therapien als integrierter Teil der Suchttherapie in ihren verschiedenen Settings. 
Viele der in der Klinik eingesetzten Verfahren sind bisher unzureichend untersucht, methodisch anspruchsvolle Studien zu psychotherapeutischen Interventionen liegen kaum vor $[8,9]$. Der Stand der Therapieforschung bezüglich der Anwendung störungsspezifischer Interventionen ist trotz ihrer klinischen Relevanz und Verbreitung unbefriedigend. Aus dem deutschsprachigen Raum wurden in den letzten Jahren kaum Studien publiziert, die hohen methodischen Standards genügen und auf der Grundlage einer Randomisierung und in Kontrollgruppendesigns durchgeführt wurden [10].

Tab. 1 zeigt die unterschiedlichen Ansätze und Blickwinkel auf, die die Vielschichtigkeit der psychosozialen Interventionen beleuchten. Nur in wenigen Arbeiten wurde jedoch vergleichend oder übergreifend geforscht, an eine Standardisierung ist vorläufig noch nicht zu denken.

So wird zum Beispiel psychiatrische Komorbidität aufgrund der Stichprobendefinition in der Untersuchungsgesamtheit des Modellversuchs Heroin eine häufige Koinzidenz darstellen. Unter anderem konnten Woody et al. [11] schon Anfang der Achtzigerjahre nachweisen, dass insbesondere diese Patientengruppen mit alleiniger Beratung unterversorgt sind und in besonderer Weise von Psychotherapien profitieren. Die Ausrichtung der Psychotherapien bei entsprechender Adaptation an das Störungsbild [9] zeigte dabei kaum Effekte.

Die Autoren überprüften u.a. vergleichend die Effekte in einem randomisierten Design an 110 Methadonpatienten und verglichen drei Therapieangebote: die traditionelle Drogenberatung „supportiv-expressiv therapy“ [12], eine psychoanalytisch orientierte Fokaltherapie, und die kognitiv-behaviorale Verhaltenstherapie [13]. Ein wesentlicher Unterschied zwischen den beiden Psychotherapiegruppen wurde nicht gefunden, wohl aber eine Überlegenheit beider der alleinigen Beratung gegenüber. Weiterhin konnten Woody et al. [11] schon früh zeigen, dass Klienten mit mittleren bis hohen Psychopathologiescores, gemessen am Addiction Severity Index [5], signifikant bessere Behandlungsergebnisse erzielten, wenn sie eine Psychotherapie erhielten.

Im Rahmen der Interpretation der Ergebnisse der großen amerikanischen Psychotherapiestudie von Kokainabhängigen [4], einer der aufwendigsten ihrer Art weltweit, verweisen die Autoren auch angesichts des Fehlens effektiver Pharmakotherapien auf die aktuelle Bedeutung der Psychotherapie- bzw. Interventionsforschung in diesem Feld.

Strain [9] fasst den Forschungsstand wie folgt zusammen: „First psychotherapy plus counceling is better than counceling alone. Second, patients with higher psychiatric severity are more likely to show benefits from psychotherapy. And third,... the benfit of psychotherapy do not extend to patients with antisocial personality disorder, except perhaps in cases has ASP and is also depressed.“

Die Kommentare des zur Würdigung der Schweizer Ergebnisse eingesetzten Reviewboards der WHO [14] verweisen auf die Notwendigkeit einer genaueren Untersuchung der Effekte der psychosozialen Therapien im Vergleich zu dem Effekt der Substanz im Rahmen eines mehrdimensionalen Behandlungsansatzes, um zur Nützlichkeit und Effektivität der medikalisierten Heroinverschreibung Stellung nehmen zu können.
Zur Bestimmung des Mehrwertes der medikalisierten Heroinverschreibung gegenüber bisherigen Behandlungsangeboten und des Stellenwertes psychosozialer und substanzbezogener Effekte ist es also notwendig, unter kontrollierten Bedingungen eine vergleichbare, standardisierte (also manualisiert vorliegende) und breit durchführbare Interventionsform $\mathrm{zu}$ wählen.

\section{Psychoedukation als Interventionsform}

Die Entwicklung, die Adaptation und der klinische Einsatz von Psychoeduktionsprogrammen erfolgt nunmehr in der Behandlung chronischer Erkrankungen seit ca. 30 Jahren [15]. Als Verfahren ist es sowohl in der Psychiatrie, der medizinischen Psychologie wie in anderen Bereichen der Medizin und Gesundheitserziehung etabliert. Die Begrifflichkeit als „Kunstbegriff“ signalisiert ihre Basis in der Lernpsychologie sowie die Nähe zur Pädagogik und zur Verhaltenstherapie $[6,16]$.

Psychoedukation hat in der Therapie chronischer, körperlicher Erkrankungen z.B. in der Behandlung des Diabetes mellitus hat eine lange Tradition. Sie ist gerade in solchen Bereichen die etablierteste psychosoziale Therapieform.

In der psychiatrischen Therapie gehört sie ebenfalls zum etablierten Angebot, besonders in der Therapie psychotischer Störungen [17]. Für dieses therapeutische Feld liegen sowohl methodisch anspruchsvolle Therapiestudien als auch elaborierte Manuale für die klinische Durchführung im deutschsprachigen Raum vor [18]. Zwar gibt es sehr spezifische Anforderungen an die Psychoedukation in der Therapie z.B. schizophrener Psychosen [17], aber es gibt auch eine Reihe Analogien und Gemeinsamkeiten mit Abhängigkeitserkrankungen. So handelt es sich bei beiden Störungsbildern um hochgradig chronische Prozesse und ein relevanter Teil der Klienten ist nicht in das bestehende Hilfesystem integriert. Über die Pharmakotherapien hinaus spielen psychosoziale Interventionen eine Schlüsselrolle für den Erfolg der Gesamttherapie [19]. Wesentliches Anliegen moderner Programme der Psychoedukation und deren besondere Stärke ist die Förderung von Lebensqualität und vorhandenen Ressourcen [20]

Der Forschungsstand zur Psychoedukation in der Drogentherapie im engeren Sinne ist derzeit unbefriedigend, beschränkt man die Referenz auf den Kernbereich der Psychoedukation $[21,22]$. Nimmt man die speziellen bzw. spezialisierten Beratungsansätze, wie das Group Counceling, hinzu [4] oder betrachtet die Angebote im Übergangsbereich von Selbsthilfe und Beratung bzw. Psychoedukation, werden interessante klinische und evaluative Parallelen deutlich, die eine gute Integration des Angebotes in der Therapie drogenabhängiger Menschen erwarten lassen.

Zusammenfassend handelt es sich bei der Psychoedukation um eine Interventionsform, die wissenschaftlich im Rahmen methodisch anspruchsvoller Studien evaluiert wurde und für die in der Behandlung chronisch Kranker mit psychischen wie somatischen Störungen viele Erfahrungen vorliegen. 
Tab.1 Aktueller Forschungsstand zu psychosozialen Interventionen in der Drogentherapie (Basis: Medlineanalyse 5.2000)

\begin{tabular}{|c|c|c|c|}
\hline Kategorien & Autor/en & Titel & Jahr \\
\hline \multirow[t]{4}{*}{ Übersichtsartikel } & $\begin{array}{l}\text { WHO-Expertengruppe zur } \\
\text { Drogenabhängigkeit }\end{array}$ & Thirtieth report. World Health Organization Technical Report Series & 1998 \\
\hline & Abdel-Mawgoud M, Fateem L et al. & Development of a comprehensive treatment program & 1995 \\
\hline & Crits-Christoph P, Siqueland L & $\begin{array}{l}\text { Psychosocial treatment for drug abuse. Selected review and } \\
\text { recommendations for national health care }\end{array}$ & 1996 \\
\hline & Siegfried N & A review of comorbidity: major mental illness and problematic substance use & 1998 \\
\hline \multirow{5}{*}{$\begin{array}{l}\text { Methadon- } \\
\text { behandlung, } \\
\text { Substitution }\end{array}$} & Alterman A, Rutherford MJ et al. & Response to methadone maintenance an counseling & 1996 \\
\hline & Belding MA, Y. Iguchi M et al. & $\begin{array}{l}\text { Assessing the helping alliance and its impact in the treatment of opiate } \\
\text { dependence }\end{array}$ & 1997 \\
\hline & Magura S, Nwakeze PC et al. & $\begin{array}{l}\text { Program quality effects on patient outcomes during methadone } \\
\text { maintenance: a study of } 17 \text { clinics }\end{array}$ & 1999 \\
\hline & Nurco DN, Primm BJ et al. & $\begin{array}{l}\text { Changes in locus-of-control attitudes about drug misuse in a self- help group } \\
\text { in a methadone maintenance clinic }\end{array}$ & 1995 \\
\hline & Scherbaum N, Heigl-Evers A & $\begin{array}{l}\text { Psychodynamische Aspekte der Substitutionsbehandlung } \\
\text { Heroinabhaengiger mit Methadon }\end{array}$ & 1996 \\
\hline \multirow[t]{4}{*}{$\begin{array}{l}\text { Ambulante } \\
\text { Behandlung }\end{array}$} & Aszalos R, McDuff DR et al. & $\begin{array}{l}\text { Engaging hospitalized heroin-dependent patients into substance abuse } \\
\text { treatment }\end{array}$ & 1999 \\
\hline & Hennessy KD, Green-Hennessy S & $\begin{array}{l}\text { An economic and clinical rationale for changing utilization review practices } \\
\text { for outpatient psychotherapy }\end{array}$ & 1997 \\
\hline & Kleinman PH, Kang SY et al. & Retention of cocaine abusers in outpatient psychotherapy & 1992 \\
\hline & Ouimette PC, Moos RH et al. & $\begin{array}{l}\text { Influence of outpatient treatment and } 12 \text {-step group involvement on } \\
\text { one-year substance abuse treatment outcomes }\end{array}$ & 1998 \\
\hline \multirow[t]{5}{*}{ Psychoedukation } & Elliott WN, Walters GD & $\begin{array}{l}\text { Conducting psychoeducational interventions with drug abusing clients: } \\
\text { the lifestyle model }\end{array}$ & 1997 \\
\hline & Ford GG & $\begin{array}{l}\text { An existential model for promoting life change. Confronting the disease } \\
\text { concept }\end{array}$ & 1996 \\
\hline & Hiller ML, Rowan-Szal GA et al. & $\begin{array}{l}\text { Effectiveness of a specialized womens intervention in a residential treatment } \\
\text { program }\end{array}$ & 1996 \\
\hline & Malow RM, West JA et al. & Outcome of psychoeducation for HIV risk reduction & 1994 \\
\hline & Sorensen JL, London J et al. & Psychoeducational group approach: HIV risk reduction in drug users & 1996 \\
\hline $\begin{array}{l}\text { Kurzinter- } \\
\text { ventionen }\end{array}$ & Baker $\mathrm{A}$, Heather $\mathrm{N}$ et al. & $\begin{array}{l}\text { Evaluation of a cognitive-behavioural intervention for HIV prevention among } \\
\text { IDU }\end{array}$ & 1993 \\
\hline \multirow{7}{*}{$\begin{array}{l}\text { (Brief-Interven } \\
\text { Motivational } \\
\text { Interviewing }\end{array}$} & ) Baker A, Kochan $\mathrm{N}$ et al. & Controlled evaluation of a brief intervention among IDU not in treatment & 1994 \\
\hline & Booth RE, Kwiatkowski C et al. & Facilitating treatment entry among out-of-treatment injection drug users & 1998 \\
\hline & Compton P, Monahan G et al. & $\begin{array}{l}\text { Motivational interviewing: an effective brief intervention for alcohol and } \\
\text { drug abuse patients }\end{array}$ & 1999 \\
\hline & Dunn CW, Ries R & $\begin{array}{l}\text { Linking substance abuse services with general medical care: integrated, brief } \\
\text { interventions with hospitalized patients }\end{array}$ & 1997 \\
\hline & Monti PM, Rohsenow DJ & $\begin{array}{l}\text { Brief coping skills treatment for cocaine abuse: substance use outcomes at } \\
\text { three months }\end{array}$ & 1997 \\
\hline & Swanson AJ, Pantalon MV et al. & $\begin{array}{l}\text { Motivational interviewing and treatment adherence among psychiatric and } \\
\text { dually diagnosed patients }\end{array}$ & 1999 \\
\hline & Werner MJ & $\begin{array}{l}\text { Principles of brief intervention for adolescent alcohol, tobacco, and other } \\
\text { drug use }\end{array}$ & 1995 \\
\hline \multirow{5}{*}{$\begin{array}{l}\text { Psychodynamische } \\
\text { und tiefen- } \\
\text { psychologische } \\
\text { Ansätze }\end{array}$} & Jerry PA & Psychodynamic psychotherapy of the intravenous cocaine abuser & 1997 \\
\hline & Markowitz JC & Developments in interpersonal psychotherapy & 1999 \\
\hline & Roback HB, Moore RF et al. & $\begin{array}{l}\text { Confidentiality dilemmas in group psychotherapy with substance- } \\
\text { dependent physicians }\end{array}$ & 1996 \\
\hline & Silvers EP & A psychotherapeutic approach to substance abuse: preliminary observations & 1993 \\
\hline & Sterling RC, Gottheil E et al. & Patient treatment choice and compliance & 1997 \\
\hline
\end{tabular}


Tab.1 Aktueller Forschungsstand zu psychosozialen Interventionen in der Drogentherapie (Basis: Medlineanalyse 5.2000)

Kategorien Autor/en Titel Jahr

Kognitive und/ oder Verhaltenstherapie

(Cognitivbehavioural therapy/CBT)
Baranackie K, Crits-Christoph P et al. Therapist techniques used during the cognitive therapy of opiate- dependent patients

Carroll KM, Schottenfeld R

Kaminer Y, Burleson JA et al.

Martinsen E, Olsen T et al.

Najavits LM, Weiss RD et al.

Simpson EB, Pistorello ] et al.

Triffleman E, Carroll K et al.

Nonpharmacologic approaches to substance abuse treatment

Psychotherapies for adolescent substance abusers: a pilot study

Cognitive-behavioral group therapy for panic disorder in the general clinical setting: a naturalistic study with 1-year follow-up

„Seeking safety“: outcome of a new cognitive-behavioral psychotherapy for women with posttraumatic stress disorder and substance dependence

Use of dialectical behavior therapy in a partial hospital program for women with borderline personality disorder

Substance dependence posttraumatic stress disorder therapy. An integrated cognitive-behavioral approach

Self-examination therapy as an adjunct treatment for depressive symptoms

1996 in substance abusing patients

reduzierung, Selbst-

beobachtung Carey KB

Carroll KM

Substance use reduction in the context of outpatient psychiatric treatment: a collaborative, motivational, harm reduction approach

Enhancing retention in clinical trials of psychosocial treatments: practical strategies

\begin{tabular}{ll}
\hline Komorbidität & Carey KB \\
& Drake RE, Antosca LM et al.
\end{tabular}

Fisher M Sr, Bentley KJ

Levy M

Links PS

Mynatt S

Nigam R, Schottenfeld R et al.

Sloan KL, Rowe G

Sonne SC, Brady KT

Treatment of co-occurring substance abuse and major mental illness

New Hampshires specialized services for the dually diagnosed

1996

Two group therapy models for clients with a dual diagnosis of substance abuse and personality disorder

Psychotherapy with dual diagnosis patients: working with denial.

Developing effective services for patients with personality disorders

Effectiveness of intervention into substance abuse disorders in women with comorbid depression

Treatment of dual diagnosis patients: a relapse prevention group approach Substance abuse and psychiatric illness: treatment experience Substance abuse and bipolar comorbidity

1996

Node-link mapping and psychological problems. Perceptions of a residential drug abuse treatment program for probationers

Accountability in public short-term adult AOD residential treatment.

Hyde A, Vollmerhausen S Inpatient treatment for substance abuse patients with psychiatric disorders:

1995 a national study of determinants of readmission

\begin{tabular}{lll}
\hline $\begin{array}{l}\text { Familientherapeu- } \\
\text { tische und } \\
\text { systemische }\end{array}$ & Diamond GS, Serrano AC et al. & Current status of family-based outcome and process research \\
Ansätze & Keller DS, Galanter M et al. & $\begin{array}{l}\text { Validation of a scale for network therapy: a technique for systematic use of } \\
\text { peer and family support in addition treatment }\end{array}$ \\
& Luthar SS, Walsh KG & $\begin{array}{l}\text { Treatment needs of drug-addicted mothers. Integrated parenting } \\
\text { psychotherapy interventions }\end{array}$
\end{tabular}

Harm reduction Rhodes F, Wolitski RJ et al.

An experiential program to reduce AIDS risk among female sex partners of

1992 injection-drug users

Vergleichende Carroll KM, Rounsaville JB et al.

Arbeiten

Carroll KM, Nich C et al.

A comparative trial of psychotherapies for ambulatory cocaine abusers: relapse prevention and interpersonal psychotherapy

Treatment of cocaine and alcohol dependence with psychotherapy and disulfiram.

Crits-Christoph P, Siqueland L et al. Kaufman E

Psychosocial treatments for cocaine dependence

Critical aspects of the psychodynamics of substance abuse and the evalua-

Pressman MA, Brook DW tion of their application to a psychotherapeutic approach

A multiple group psychotherapy approach to adolescents with psychiatric and substance abuse comorbidity

Woody GE, McLellan AT et al.

\section{hr}

\section{2}

6

6


Tab.1 Aktueller Forschungsstand zu psychosozialen Interventionen in der Drogentherapie (Basis: Medlineanalyse 5.2000)

\begin{tabular}{lll}
\hline Kategorien & Autor/en & Titel \\
\hline Einzelne Themen & Ball SA, Legow NE & $\begin{array}{l}\text { Attachment theory as a working model for the therapist transitioning from } \\
\text { early to later recovery substance abuse treatment } \\
\text { Motivation and readiness for therapeutic community treatment among } \\
\text { cocaine and other drug abusers }\end{array}$ \\
& Ge Leon G, Melnick G et al. & $\begin{array}{l}\text { Homelessness and mental illness in a professional- and peer-led cocaine } \\
\text { treatment clinic }\end{array}$ \\
Hodgins DC, el-Guebaly N et al. & $\begin{array}{l}\text { Treatment of substance abusers: single or mixed gender programs? } \\
\text { Hoffman JA, Caudill BD et al. }\end{array}$ & $\begin{array}{l}\text { Psychosocial treatments for cocaine abuse. } 12 \text {-month treatment outcomes } \\
\text { Increasing adherence to substance abuse aftercare group therapy } \\
\text { Lash SJ, Blosser SL }\end{array}$ \\
Shaffer HJ, LaSalvia TA et al. & $\begin{array}{l}\text { Comparing Hatha yoga with dynamic group psychotherapy for enhancing } \\
\text { methadone maintenance treatment: a randomized clinical trial }\end{array}$
\end{tabular}

\section{Diskussion des Einsatzes der Psychoedukation}

Es gibt Versuche mit allen Formen von Psychotherapie in allen Settings, ohne dass sich wissenschaftlich evaluierte Standards oder Leitverfahren entwickelt hätten $[8,23]$. In der Psychiatrie sind die Gestalttherapie und die humanistische Psychologie [23] häufiger vertreten. In der Behandlung vor allem von Alkoholabhängigkeit spielt die Verhaltenstherapie eine wichtige Rolle [13].

An ein psychotherapeutisches Verfahren sind auf der Grundlage der Voraussetzungen im Hilfesystem heute folgende Anforderungen zu stellen. Diese gelten analog auch für die psychosozialen Betreuungsstandards im Modellprojekt:

1. Das Verfahren muss realisierbar sein, ohne dass die Durchführenden eine der etablierten Therapieausbildungen absolviert haben, um es möglichst vielen Berufsgruppen zugänglich zu machen.

2. Dabei muss es so weit wie möglich standardisierbar sein, um gleiche Standards in der Behandlung zu sichern und so weitgehende Vergleichbarkeit der Interventionen im Rahmen von Therapieforschung sicherzustellen.

3. Es muss in einem überschaubaren zeitlichen Rahmen zu messbaren Effekten hinsichtlich der Wirkungserwartungen führen.

4. Die praktischen Erfahrungen aus dem bestehenden Hilfesystem sollten möglichst optimal in das Programm integrierbar sein, auch um gute Grundlagen für die Weiterentwicklung zu ermöglichen.

5. Der notwendige Qualifikationsaufwand sollte überschaubar und in der zur Verfügung stehenden Zeit realisierbar sein.

6. Das Verfahren sollte die Basis und einen geeigneten Rahmen für eine Adaptation an die Erfordernisse des Modellversuches und die zu behandelnde Klientel bieten.

Die wesentlichen Gründe, die dazu geführt haben, auf diesem Hintergrund die Psychoedukation als Verfahren zu erwägen, sind die folgenden:

- Positive Erfahrungen in der Therapie chronischer Erkrankungen insbesondere von Psychosen [17] und chronisch körperlichen Erkrankungen kennzeichnen die Psychoedukation als Verfahren, das gut standardisierbar ist und bestehende vielfältige Behandlungsprogramme wirkungsvoll ergänzt. Es hat seine Effektivität in der Behandlung im Rahmen klinischer Prüfungen unter Beweis gestellt [16].
- Die patientenbezogenen Anforderungen an psychosoziale Interventionen in der Drogentherapie sind gerade angesichts einer mehrfach beeinträchtigten Klientel komplex. Die in der Psychoedukation strukturierte Vorgehensweise und vergleichbare Vorgehensweisen in der Selbsthilfe und der Beratung [24] lassen erwarten, dass sie einen effektiven Rahmen bieten und die Anknüpfungspunkte in der bestehenden Beratungstätigkeit optimal nutzen können. Praktische Erfahrungen im Rahmen der Methadonsubstitution in der Schweiz und den USA bestätigen diese Erwartung.

- Eine niedrigschwellige Einsetzbarkeit durch verschiedene Berufsgruppen ohne die Notwendigkeit einschlägiger Therapieausbildungen ist als Voraussetzung für einen breiten Einsatz im Hilfesystem mit den Erfahrungen in der Psychosentherapie belegt. Der zu erwartende Aufwand liegt in einem kalkulierbaren zeitlichen Rahmen.

- Neben der Bearbeitung von Defiziten und Risiken bietet die Psychoedukation die Möglichkeit, eine Ressourcenorientierung in den Programmen zu verankern [20]. Sie böte so die Möglichkeit zur Integration bestehender Interventionsstrategien aufgrund der Flexibilität der Interventionsform.

Es gibt indes wenig evaluierte Erfahrungen zur Psychoedukation aus der Drogenhilfe; das Gros der Erfahrungen stammt aus anderen Bereichen. Daraus ergibt sich bei der Planung ein hoher Vorbereitungsaufwand (Manualisierung, Machbarkeitsstudie) und die Notwendigkeit der Adaptation an die Bedingungen der Drogenhilfe.

\section{Schritte zu Manualisierung und Machbarkeit}

Gesamtstruktur und Philosophie, die Ziele und Schwerpunkte allgemein sowie die einzelnen Sitzungen müssen operationalisiert werden. Entsprechende Hilfsmittel und Anschauungsmaterialien sollten zur Verfügung gestellt werden. Die Erstellung des Materials wird dabei die Erfahrungen aus der Psychotherapieforschung allgemein und aus der Drogenhilfe speziell berücksichtigen.

Einer der ersten Schritte neben der Adaptation der allgemeinen Prinzipien ist die Selektion der zu behandelnden Themenbereiche. $\mathrm{Zu}$ behandelnde Bereiche sollten sein:

- Risikomanagement, Erkennen von Risikofaktoren, Verhalten bei Überdosierungen

- Rückfallmanagement

- psychische Krankheiten und Sucht - Suizidalität, Depression, Angst, Psychose, Delir 
- Kommunikationskompetenz

- Familie und soziales Netz

- körperliche Gesundheit und Krankheit

- Strategien zur Erhöhung der Lebensqualität (analog des Wiener Psychoedukationsprogramms), Ressourcen heben, Lebensqualität verbessern

- Therapiebedarf und Therapiemöglichkeiten

Die Eckpunkte der Interventionen können analog der bekannten Psychoedukationsprogramme adaptiert werden.

Dies bedeutet:

1. Vorbereitende Qualifikation der Durchführenden durch einführende Seminare.

2. Die Motivation der Teilnehmer erfolgt durch zielgruppenspezifische Informationsmaterialien, persönliche Information und Motivation durch die Drogenberater.

3. Die Durchführung sollte analog der Erfahrungen in anderen Bereichen in Gruppen erfolgen.

4. Die Dauer der Intervention beträgt 12-15 Sitzungen, bei einer Sitzung pro Woche.

5. Zur Unterstützung eines kontinuierlichen Prozesses und der Vertiefung der Ergebnisse kann danach einmal monatlich eine Auffrischungssitzung erfolgen.

6. Die Qualitätssicherung der Intervention sollte durch unterschiedliche begleitende Maßnahmen der Dokumentation, Teilnehmer und Gruppenleiterbefragung erfolgen.

\section{Abschließende Bemerkung}

Zusammenfassend stehen der Suchttherapie neben der Entwicklung in der Psychopharmakotherapie auch interessante Innovationen in der Ausgestaltung psychosozialer Interventionen bevor. Die Entwicklung und Adaptation von Psychoedukation in der Drogentherapie ist unter verschiedenen Aspekten ein vielversprechender Ansatz.

Vielleicht kann der Modellversuch der heroingestützten Behandlung darüber hinaus auch auf diesem Wege zur Innovation und Qualifikation der psychosozialen Betreuung sowie der Therapieforschung beitragen.

\section{Literatur}

${ }^{1}$ Bühringer G, Gastpar M, Heinz W, Kovar KA, Ladewig D, Naber D, Täschner KL, Uchtenhagen A, Wanke K. Methadon-Standards. Vorschläge zur Qualitätssicherung bei der Methadon-Substitution im Rahmen der Behandlung von Drogenabhängigen. Stuttgart: Enke, 1995

2 American Psychiatric Association (APA). Practice guideline for the treatment of patients with substance use disorders: alcohol, cocaine, opioids. Am J Psychiatry 1995; 152 (suppl): 5-59

${ }^{3}$ Leitlinien für die psycho-soziale Begleitung im Rahmen einer Substitutionsbehandlung. Akzept (Hrsg). 1995

${ }^{4}$ Crits-Christoph P, Siqueland L, Blaine J, Frank A, Luborsky L, Onken LS, Muenz LR, Thase ME, Weiss RD, Gastfriend DR, Woody GE, Barber JP, Butler SF, Daley D, Salloum I, Bishop S, Najavits LM, Lis J, Mercer D, Griffin ML, Moras K, Beck AT. Psychosocial treatments for cocaine dependence: National Institute on Drug Abuse Collaborative Cocaine Treatment Study (see comments). Archives Of General Psychiatry 1999; 56 (6): 493-502

${ }^{5}$ McLellan AT, Arndt IO, Metzger DS, Woody GE, OBrien CP. The effects of psychosocial services in substance abuse treatment. Journal of the American Medical Association 1993; 269: 1953-1959
${ }^{6}$ Lowinson JH, Ruiz P, Millmann RB, Langrod JG. Substance Abuse, A Comprehensive Textbook. Baltimore: Williams and Wilkins, 3rd Ed, 1997

7 Woody GE, McLellan A, Luborsky L, OBrien CP. Psychotherapy and counseling for methadone-maintained opiate addicts: Results of research studies. Nida Res Monogr Ser 1990; 104 9-23

${ }^{8}$ Grawe K, Donati R, Bernauer F. Psychotherapie - Von der Konfession zur Profession? Weinheim: Beltz, 1993

${ }^{9}$ Strain EC. Psychosocial Treatments for Cocaine Dependance. Arch Gen Psychiatry 1999; 56 (6): 503-504

${ }^{10}$ Ladewig D, Battegay I, Stohler R, Erb P, Rohr HP, Uchtenhagen A, Gyr K. A randomised trial with methadone, morphine and heroin in the treatment of opiate dependence. 1997

11 Woody GE, McLellan AT, Luborsky L, O'Brien CP, Blaine J, Fox S, Hermann I, Beck AT. Psychiatric severity as a predictor of benefits from psychotherapy: The Penn-VA study. Am J Psychiatry 1984; 141 (10): 1172-1177

12 Luborsky L, Woody GE, Hole AV, Velleco A. Treatment Manual for Supportive - Expressive Dynamic Psychoanalytically oriented Special Adaptation for Treatment of Drug Dependence. Philadelphia: University of Pennsylvania, 1989

13 Carroll KM, Nich C, Ball SA, McCance E, Rounsavile BJ. Treatment of cocaine and alcohol dependence with psychotherapy and disulfiram. Addiction 1998; 93 (5): 713-27

${ }^{14}$ WHO. Report of the external panel on the evaluation of the Swiss scientific studies of medically prescribed narcotics to drug addicts. Genf: 1999

15 Liberman, Mueser K, Wallace CJ, Jacobs HE, Eckmann T et al. Training skills in the severely psychiatrically disabled: learning coping and competence. Schizophrenia Bulletin 1986; 12: 631641

${ }^{16}$ Hornung WP. Psychoedukation und Psychopharmakotherapie. Zur Kooperation schizophrener Patienten. Stuttgart: Schattauer, 1998

${ }^{17}$ Hornung WP. Psychoedukative Interventionen. In: Krausz M\& Naber D (Hrsg). Integrative Schizophrenietherapie, Basel: Karger, 2000: 113-147

18 Wienberg G. Schizophrenie zum Thema machen. Bonn: Psychiatrie Verlag, 1995

19 Krausz M. Integrative Schizophrenietherapie. Behandlungsphilosophie und Interventionen. Naber D (Hrsg). Basel: Karger, 2000

${ }^{20}$ Amering. 1998 Abstract, Kongressband, „Die subjektive Seite der Schizophrenie“. Hamburg: 1999

${ }^{21}$ Liese BS. Brief Therapy, crisis intervention, and the cognitive therapy of substance abuse. Crisis Intervention and Time limited Treatment. 1994; 1: 11-29

22 Wetzler S, Schwarzt BJ, Sanderson W, Karasu TB. Academic psychiatry and managed care: a case study. Psychiatr Serv Aug1997; 48 (8): 1019-26

23 Petzold H, Scheiblich W, Thomas G. Psychotherapeutische Maßnahmen bei Drogenabhängigen. In: Uchtenhagen, A, Zieglgänsberger W (Hrsg). Suchtmedizin, München, Jena: Urban und Fischer, 2000

${ }^{24}$ Mercer DE, Woody GE. Individual Drug Counceling (IDC) Treatment Manual. Philadelphia: University of Pennsylvania, 1999

\section{Michael Krausz, Georg Farnbacher}

Zentrum für Interdisziplinäre Suchtforschung der Universität Hamburg

c/o Zentrum für psychosoziale Medizin, Psychiatrie und Psychotherapie im UKE

Martinistraße 52

20246 Hamburg

E-mail: krausz@uke.uni-hamburg.de 